\title{
Nuclear structures: Twinning and modulation in crystals
}

\author{
Václav Petř́ček and Michal Dušek \\ Institute of Physics, Czech Academy of Sciences v.v.i., Na Slovance 2, 182 21Praha 8, Czech Republic
}

\begin{abstract}
Crystal structure analysis is a standard technique routinely applied to single crystals as well as powders. However the process is not so straightforward if the crystal sample is affected by twinning or if the structure is modulated. In such cases the standard procedures are not directly applicable. The main purpose of this contribution is to show how to solve and refine such difficult structures. While for twinned structures the basic property of crystal - translation symmetry in three dimensional space-remains valid, for modulated crystals a special superspace theory must be exploited in order to describe the atomic structure with crystallographic methods generalized for superspace.
\end{abstract}

\section{Introduction}

Modern structure analysis of single crystals based on diffraction experiment is nowadays a standard discipline which allows solving and refining structures of most new crystalline materials. Data are usually acquired on laboratory X-ray diffractometers, and structure solution and refinement is made with standard program packages such SHELX [1], Olex2 [2], Crystals [3] or Jana2006 [4] in almost automatic way. Such a work can be made by a nonspecialist in crystallography because most of possible problems can be detected and corrected with help of checking program such as Platon [5]. Simple structure of a well diffracting sample can be now measured, solved and refined in less than one hour and the task can be done without a knowledge what is behind these powerful tools.

However, not all structures can be solved by using these standard approaches. Two effects - twinning and modulations - often make serious problems in structure solution and refinement if they are present in the crystals. These effects are especially important during phase transitions where electric and/or magnetic properties of the studied crystal may depend substantially on the phase and therefore a full description of the corresponding structural changes is very important. If these changes affect atomic positions as well as ordering of magnetic moments, such crystals must be studied by both X-ray and neutron diffraction technique. In this paper we shall concentrate on problems of solution and refinement of nuclear structure from the neutron diffraction experiment. However, most of explanations are also valid for X-ray diffraction.

In spite of the fact that most of standard programs can handle twinning in the crystal, application of these tools needs much deeper understanding of crystallography. Even more complicated are modulated crystals, which miss the basic property of the classical crystal, i.e. translation symmetry. In this paper we will introduce shortly the problem of solution and refinement of difficult structures. For more details about the underlying theory, we recommend the monographs [6] and [7]. 




Figure 1. Diffraction pattern of monoclinic crystal, with monoclinic angle $\beta$ different for $90^{\circ}$, twinned by a general rotation $180^{\circ}$ along $\mathrm{c}$ axis.

\section{Twinning}

\subsection{Overlaps in twinned crystals}

Twinned crystals are supposed to be composed from structurally identical domains mutually related by proper or improper rotations. The number of different domains is usually not very large and in most cases there are present just two differently oriented domains. The simplest case leads to existence of two mutually rotated diffraction patterns which can be simply recognized from diffraction pattern, see Fig. 1.

In this example, reflections $(h, k, 0)$ are systematically overlapped with reflections $(-h,-k, 0)$ of the second domain. Depending on the monoclinic angle $\beta$, additional (random) overlapping reflections may occur for $(h, k, l)$ with $l$ being close to $2 n a / c \cos \beta$.

The relationship between orientations of two domains is related by so called twinning matrix $\mathbf{T}_{2}$ which is defined by the equation:

$$
\left[\begin{array}{lll}
\mathbf{a}_{1} & \mathbf{b}_{1} & \mathbf{c}_{1}
\end{array}\right]=\left[\begin{array}{lll}
\mathbf{a}_{2} & \mathbf{b}_{2} & \mathbf{c}_{2}
\end{array}\right] \cdot \mathbf{T}_{2}
$$

where $\mathbf{a}_{i}, \mathbf{b}_{i}$ and $\mathbf{c}_{i}$ are cell parameters of the $\mathrm{i}^{\text {th }}$ domain. The twinning matrix can be as used to express diffraction indices of the second domain in the reciprocal coordinate system of the first (reference) system:

$$
\left[\begin{array}{lll}
h_{21} & k_{21} & l_{21}
\end{array}\right]=\left[\begin{array}{lll}
h_{2} & k_{2} & l_{2}
\end{array}\right] \cdot \mathbf{T}_{2}
$$

While the indices $\left(h_{2}, k_{2}, l_{2}\right)$ are integers defining some reflections from the second domain, their corresponding coordinates $\left(h_{21}, k_{21}, l_{21}\right)$ in the reference subsystem of the first domain need not to be integers. The twinning matrix can be used for predicting the full and partial 


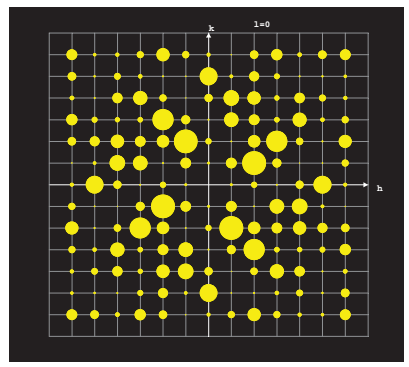

(a)

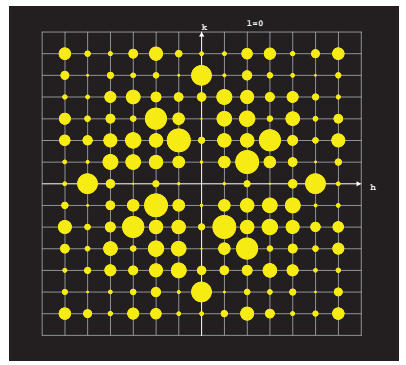

(b)

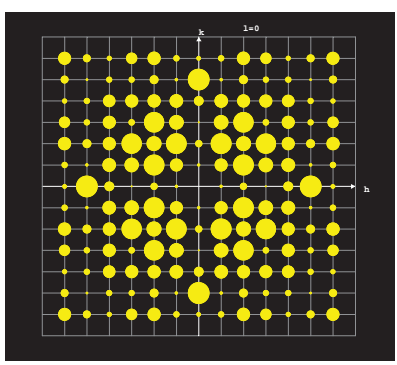

(c)

Figure 2. $(h, k, 0)$ diffraction plane of a simulated structure with point group 4/m: (a) without twinning; (b) twinned by $180^{\circ}$ rotation along b axis with volume fractions (3:1); (c) twinned by $180^{\circ}$ rotation along $\mathrm{b}$ axis with volume fractions $(1: 1)$.

overlaps of diffraction spots, using the distance in reciprocal space between $\left(h_{21}, k_{21}, l_{21}\right)$ and the closest $\left(h_{1}, h_{1}, l_{1}\right)$. The reflections are supposed to be fully overlapped if their distance is smaller than the resolution limit of the data collection and fully separated if the distance is larger than the selected limit for such separation. Reflections having partial overlaps, i.e. with their distance larger than the resolution limit but smaller than the limit for full separation should be discarded from refinement.

Such a criterion is very rough and it might lead to relatively large number of deleted reflections. Moreover, the resolution and separation limits are not the same for all pairs of reflections, because they may be registered in an area detector at different geometry. For this reason, the standard data processing programs like Crysalis, Saint or Eval recognize the overlaps during data processing and encode corresponding information (i.e. which reflections are overlapped and separated) in a form of so-called hklf5 format. In such case the partially overlapped reflections are considered together in the refinement and no information needs to be deleted.

The case presented in the Fig. 1 is simple: the twinning is easily recognized from the diffraction pattern, the structure is solved by standard techniques from one domain or from a detwinned data and, finally, the structure is refined using the twinning matrix or hklf5 format.

Twins with full overlap of all reflections are much more difficult case since they cannot be easily recognized. In the Fig. 2 we present a simulated diffraction pattern of a tetragonal structure with point symmetry $4 / \mathrm{m}$, having different portion of twin domains related by $180^{\circ}$ rotation along b axis. In cases (b) and (c) no indication of twinning is apparent and, moreover, the symmetry of the diffraction pattern (c) is higher $(4 / \mathrm{mmm})$ than the Laue symmetry of the structure. This means that presence of twinning in the crystal may obscure the correct symmetry, which can be deduced only during solution and refinement process. From Fig. 2, the necessary condition for such full overlaps is obvious: the point symmetry of the reciprocal lattice must be higher than the point symmetry of the structure.

Similar effects can be present even for structures of lower symmetry (triclinic, monoclinic, ...) if their cell parameters correspond within the experimental accuracy to a higher lattice point symmetry. This may happen as a result phase transitions in which the original symmetry is reduced but cell parameters are almost unchanged. In the following we shall concentrate on problems of data processing, solution and refinement of twins with full overlaps. 


\subsection{Symmetry of the twinned diffraction pattern}

Overlapping of diffraction spots from $n$ twin domains can be expressed by the following formula for the combined structure factor $\mathcal{F}(\mathbf{h})$ :

$$
\mathcal{F}^{2}(\mathbf{h})=v_{1} F^{2}\left(\mathbf{h} \mathbf{T}_{1}\right)+v_{2} F^{2}\left(\mathbf{h} \mathbf{T}_{2}\right)+\ldots+v_{n} F^{2}\left(\mathbf{h} \mathbf{T}_{n}\right)
$$

where $v_{i}$ is the volume fraction of $\mathrm{i}^{\text {th }}$ domain and $F(\mathbf{h})$ is the structure factor. The volume fractions are normalized to 1 :

$$
\sum_{i=1}^{n} v_{i}=1
$$

The Eq. (3) is based on the assumption that diffractions from twin domains are independent. The structure factors $F(\mathbf{h})$ are coefficients in the Fourier summation of periodic density of scatterers $\rho(\mathbf{r})$ :

$$
\begin{gathered}
\rho(\mathbf{r})=\rho\left(\mathbf{r}+\sum_{i=1}^{3} n_{i} \mathbf{a}_{i}\right) \\
\rho(\mathbf{r})=\sum_{\mathbf{h}} F(\mathbf{h}) \exp (-2 \pi i \mathbf{h r})
\end{gathered}
$$

The combined structure factor is $\mathcal{F}(\mathbf{h})$ and it can be calculated from the integrated intensity $I(\mathbf{h})$ of the relevant diffraction spot as for a non-twinned crystal:

$$
I(\mathbf{h})=S \cdot L p(|\mathbf{h}|) \cdot A(\mathbf{h}) \cdot \mathcal{F}^{2}(\mathbf{h})
$$

where $S$ stands for the scaling factor, A for absorption correction and $L p$ for Lorentzpolarization factor.

The full symmetry of the crystal is expressed by the space group symmetry which consists from the symmetry operations of the type:

$$
\mathbf{r}^{\prime}=\mathbf{R}_{j} \mathbf{r}+\mathbf{s}_{j}+\mathbf{c}_{k}+\mathbf{n}
$$

where $\mathbf{R}_{i}$ and $\mathbf{s}_{i}$ are rotation and translation part of the $\mathrm{i}^{\text {th }}$ symmetry operation, $\mathbf{c}_{j}$ is $\mathrm{j}^{\text {th }}$ centering vector and $\mathbf{n}$ is an arbitrary unit cell translation. Then the generalized equation for symmetry condition is:

$$
\rho(\mathbf{r})=\rho\left(\mathbf{R}_{j} \mathbf{r}+\sum_{i=1}^{3}\left(s_{j i}+c_{k i}+n_{i}\right) \mathbf{a}_{i}\right)
$$

From the Eqs. (9) and (6) we can get:

$$
F\left(\mathbf{h} \mathbf{R}_{j}\right)=F(\mathbf{h}) \exp \left[2 \pi i \mathbf{h}\left(\mathbf{s}_{j}+\mathbf{c}_{k}\right)\right]
$$

and for squared structure factors the following symmetry relationship holds:

$$
F^{2}\left(\mathbf{h} \mathbf{R}_{j}\right)=F^{2}(\mathbf{h})
$$

This means that the diffraction pattern follows the point group symmetry. Moreover, the fact that the nuclear density is a real function leads to the following relationship for the complex conjugated structure factor:

$$
F^{*}(\mathbf{h})=F(-\mathbf{h})
$$

and therefore the diffraction pattern shows contains always an inversion center even for noncentrosymmetric structures. 
As mentioned above, complete overlaps of diffraction spots of the twinned crystals are possible only if the point symmetry of the lattice $\mathcal{H}$ is higher than the point symmetry of the structure $\mathcal{G}$. Say that the order of the subgroup $\mathcal{G}$ with respect to $\mathcal{H}$ is $\mathrm{n}$. Then the point group $\mathcal{H}$ can be decomposed into left cosets:

$$
\mathcal{H}=\mathbf{T}_{1} \mathcal{G}+\mathbf{T}_{2} \mathcal{G}+\ldots+\mathbf{T}_{n} \mathcal{G}
$$

where the set of twinning operations $\left\{\mathbf{T}_{1}, \mathbf{T}_{2}, \ldots . \mathbf{T}_{n}\right\}$ are selected arbitrary as representatives of each coset. Without loss of generality the first twinning element can be chosen as an identity operation.

Symmetry of the diffraction pattern in the case of equal volume fractions follows the lattice symmetry $\mathcal{H}$. But as volume fractions need not be exactly equal we cannot a priory use during data merging all symmetry operations from $\mathcal{H}$. Question is if all symmetry operations from the point group $\mathcal{G}$ are always present in the diffraction pattern. For the reflection $\mathbf{h G}_{j}$ where $\mathbf{G}_{j}$ is an arbitrary operation from $\mathcal{G}$ the Eq. (3) gives:

$$
\mathcal{F}^{2}\left(\mathbf{h G} \mathbf{G}_{j}\right)=v_{1} F^{2}\left(\mathbf{h} \mathbf{G}_{j} \mathbf{T}_{1}\right)+v_{2} F^{2}\left(\mathbf{h} \mathbf{G}_{j} \mathbf{T}_{2}\right)+\ldots+v_{n} F^{2}\left(\mathbf{h} \mathbf{G}_{j} \mathbf{T}_{n}\right)
$$

then the symmetry requirement $\mathcal{F}^{2}\left(\mathbf{h G}_{j}\right)=\mathcal{F}^{2}(\mathbf{h})$ leads to set of conditions:

$$
\mathbf{G}_{j} \mathbf{T}_{k}=\mathbf{T}_{k} \mathbf{G}_{l} \Rightarrow \mathbf{G}_{l}=\mathbf{T}_{k}^{-1} \mathbf{G}_{j} \mathbf{T}_{k}
$$

where $\mathbf{G}_{l}$ is again an operation from $\mathcal{G}$. These conditions are fulfilled for a subgroup $\mathcal{G}$ only if it is a normal subgroup in $\mathcal{H}$. In case the subgroup $\mathcal{G}$ is not a normal subgroup of $H$ only operations fulfilling the Eq. (14) can be used in the merging procedure of symmetry equivalent reflections. Note that if the order of the subgroup $\mathcal{G}$ in $\mathcal{H}$ is two, the subgroup is always normal.

The conclusion about symmetry of the diffraction pattern is that the symmetry can mimic the lattice symmetry but it can even mimic a symmetry lower than the point group of the crystal.

\subsection{Systematic extinctions of twins}

Systematic extinctions are used to determine a correct space group of the studied compound. From the Eq. (10) it follows that reflections fulfilling the condition $\mathbf{h} \mathbf{R}_{j}=\mathbf{h}$ (i.e. $\mathbf{h}$ invariant with respect to $\mathbf{R}_{j}$ ) can have non-zero structure factor only if:

$$
\exp \left[2 \pi i \mathbf{h}\left(\mathbf{s}_{j}+\mathbf{c}_{k}\right)\right]=1 \quad \Rightarrow \quad \mathbf{h}\left(\mathbf{s}_{j}+\mathbf{c}_{k}\right)=n
$$

where $n$ is an arbitrary integer.

Example: The mirror plane with its normal parallel to the $\mathbf{c}$ axis has the following matrix form with respect to the crystal coordinate system $\mathbf{a}, \mathbf{b}$ and $\mathbf{c}$ :

$$
\left(\left[\begin{array}{ccc}
1 & 0 & 0 \\
0 & 1 & 0 \\
0 & 0 & -1
\end{array}\right],\left[\begin{array}{l}
s_{1} \\
s_{2} \\
s_{3}
\end{array}\right]\right)
$$

From the space group theory it follows that in a primitive unit cell $s_{1}$ and $s_{2}$ are equal either to 0 or $1 / 2$ and that the third component $s_{3}$ in not restricted and depends on the origin selection. Taking into account all combinations and the Eq. (15), we can get extinctions as summarized in the Table 1. 
Table 1. Systematic extinctions for $m(001)$.

\begin{tabular}{|l|c|c|}
\hline$\left(\boldsymbol{s}_{\mathbf{1}}, \boldsymbol{s}_{\mathbf{2}}, \boldsymbol{s}_{\mathbf{3}}\right)$ & Symmetry operation & Extinction condition \\
\hline$(0,0,0)$ & $\mathrm{m}$ & - \\
\hline$(1 / 2,0,0)$ & $\mathrm{a}$ & $h=2 n+1$ \\
\hline$(0,1 / 2,0)$ & $\mathrm{b}$ & $k=2 n+1$ \\
\hline$(1 / 2,1 / 2,0)$ & $\mathrm{n}$ & $h+k=2 n+1$ \\
\hline
\end{tabular}

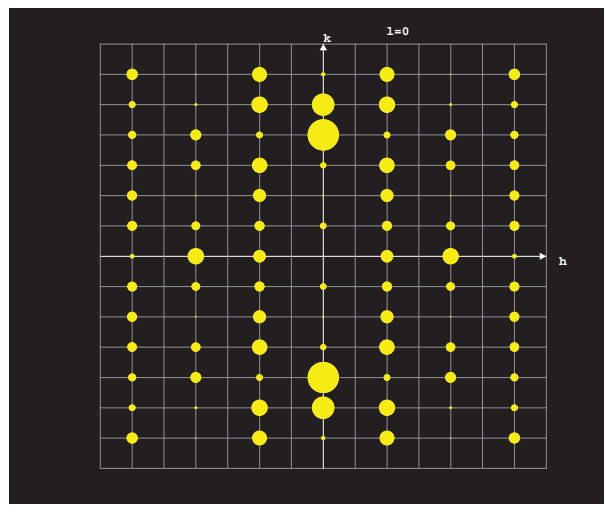

(a)

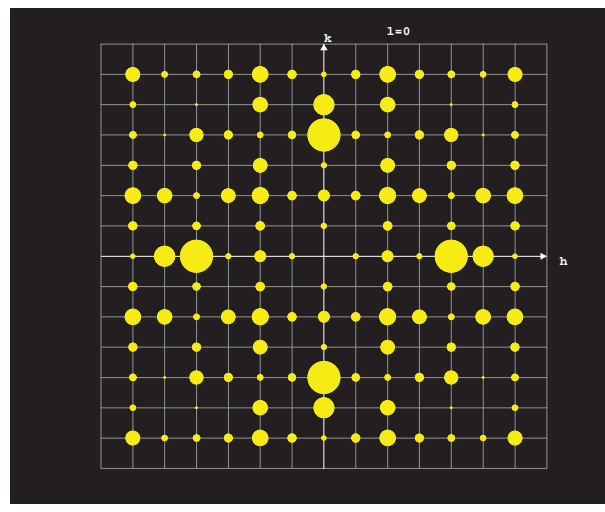

(b)

Figure 3. $(h, k, 0)$ diffraction plane of simulated structure with an a-glide plane: (a) without twinning; (b) twinned by $90^{\circ}$ rotation around c axis with volume fractions $1: 1$.

Overlapping of reflections in twinned crystals can partially hide the extinction conditions. In the example shown in the Fig. 3 such an effect is demonstrated for the a-glide plane:

As a result of the twinning, the absent reflections must fulfill the conditions $h=2 n+1$ and $k=2 n+1$, which cannot occur for a non-twinned crystal. Thus the extinctions are violated in very specific way which can help to recognize that the crystal in twinned.

\subsection{Solution and refinement of twinned structures}

Solution of crystal structures of twinned samples with completely overlapping diffraction patterns may be a difficult task. In cases where twinning is a result of a phase transition from higher to lower symmetrical phase, the known structural model of the higher symmetrical phase can be used as a starting point for the structural solution of the lower symmetrical phase. However, in many cases several different subgroups are to be tested to find correct solution.

In the case that domains are not equally occupied and an approximate volume fraction is known, the observed structure factors can be corrected to get "detwinned" structure factors useful for ab-initio solution by standard methods:

$$
F^{2}(\mathbf{h})=\left[(1-v) \mathcal{F}^{2}(\mathbf{h})-v \mathcal{F}^{2}\left(\mathbf{h} \cdot \mathbf{T}_{2}\right)\right] /(1-2 v)
$$

However, this correction cannot be used for equally or almost equally occupied domains.

On the other hand, as soon as some starting structural model is known, the refinement and completing the structure is very similar to that of a regular structure. The number of parameters in the refinement is enlarged only by the (n-1) volume fractions. 
For completing the structure a Fourier maps can be used in which the observed structure factors are corrected for twinning by one of these methods:

$$
\begin{gathered}
F_{\text {obs }, \text { corr }}^{2}(\mathbf{h})=\frac{1}{v_{1}}\left[\mathcal{F}_{\text {obs }}^{2}(\mathbf{h})-v_{2} F_{\text {calc }}^{2}\left(\mathbf{h} \cdot \mathbf{T}_{2}\right)-v_{3} F_{\text {calc }}^{2}\left(\mathbf{h} \cdot \mathbf{T}_{3}\right)-\ldots-v_{n} F_{\text {calc }}^{2}\left(\mathbf{h} \cdot \mathbf{T}_{n}\right)\right] \\
\left|F_{\text {obs }, \text { corr }}(\mathbf{h})\right|=\left|\mathcal{F}_{\text {obs }}(\mathbf{h})\right| \frac{\left|F_{\text {calc }}(\mathbf{h})\right|}{\left.\mid \mathcal{F}_{\text {calc }} \mathbf{h}\right) \mid}
\end{gathered}
$$

The above described methods handling twinned crystals have been implemented in the program Jana2006 and several typical practical examples were included into Jana cookbook (examples 3.1-3.4). Both can be downloaded the Jana home page: http://jana.fzu.cz/.

\section{Modulated crystals}

\subsection{Superspace approach for modulated structures}

Translational periodicity is the basic property of the classical crystal, see the Eq. (5). A direct consequence of this periodicity is the existence of a diffraction pattern by X-ray, neutrons or electrons with sharp diffraction spots which are localized at the points of the reciprocal lattice defined by basic vectors $\mathbf{a}_{1}^{*}, \mathbf{a}_{2}^{*}$ and $\mathbf{a}_{3}^{*}$ :

$$
\mathbf{h}=h_{1} \mathbf{a}_{1}^{*}+h_{2} \mathbf{a}_{2}^{*}+h_{3} \mathbf{a}_{3}^{*}
$$

The reciprocal lattice vectors are related to translational (direct space) lattice vectors by equations:

$$
\mathbf{a}_{1}^{*}=\frac{\mathbf{a}_{2} \times \mathbf{a}_{3}}{\mathbf{a}_{1}\left(\mathbf{a}_{2} \times \mathbf{a}_{3}\right)} \quad \mathbf{a}_{2}^{*}=\frac{\mathbf{a}_{3} \times \mathbf{a}_{1}}{\mathbf{a}_{1}\left(\mathbf{a}_{2} \times \mathbf{a}_{3}\right)} \quad \mathbf{a}_{3}^{*}=\frac{\mathbf{a}_{1} \times \mathbf{a}_{2}}{\mathbf{a}_{1}\left(\mathbf{a}_{2} \times \mathbf{a}_{3}\right)}
$$

However, there are compounds which also give additional sharp diffraction spots, so-called satellites, localized out of the nodes of the reciprocal lattice. These spots can be indexed only if one or more additional (so called modulation) vectors are used:

$$
\mathbf{H}=h_{1} \mathbf{a}_{1}^{*}+h_{2} \mathbf{a}_{2}^{*}+h_{3} \mathbf{a}_{3}^{*}+m \mathbf{q} \ldots
$$

In most cases one modulation vector is sufficient for indexing of satellites, and in the following text we shall confine ourselves to the case with one modulation vector. The diffraction pattern of such modulated crystal looks like the one shown in the Fig. 4.

The fact that the additional (satellite) reflections are sharp and regularly distributed in the diffraction space means that the violation of the $3 \mathrm{~d}$ translation symmetry must be somehow regular. For handling of modulated structure a special theory of superspace and its symmetry has been developed. The development is closely connected with names Peter de Wolff, Aloysio Janner and Ted Janssen [8].

The superspace approach is based on the construction which moves artificially all satellite reflections into the $4^{\text {th }}$ dimension with a shift proportional to the satellite index - see Fig. 5 . The spots are now making a lattice in the four dimensional reciprocal space described by the reciprocal vectors:

$$
\mathbf{A}_{i}^{*}=\mathbf{a}_{i}^{*} \quad \text { for } i=1,2,3 \quad \mathbf{A}_{4}^{*}=\mathbf{q}+\mathbf{e}
$$

The lattice character of the diffraction pattern in $(3+1) \mathrm{d}$ superspace means that the modulated structure has a translation periodicity in $(3+1) \mathrm{d}$ superspace. Thus the generalized 


\section{JDN 22}

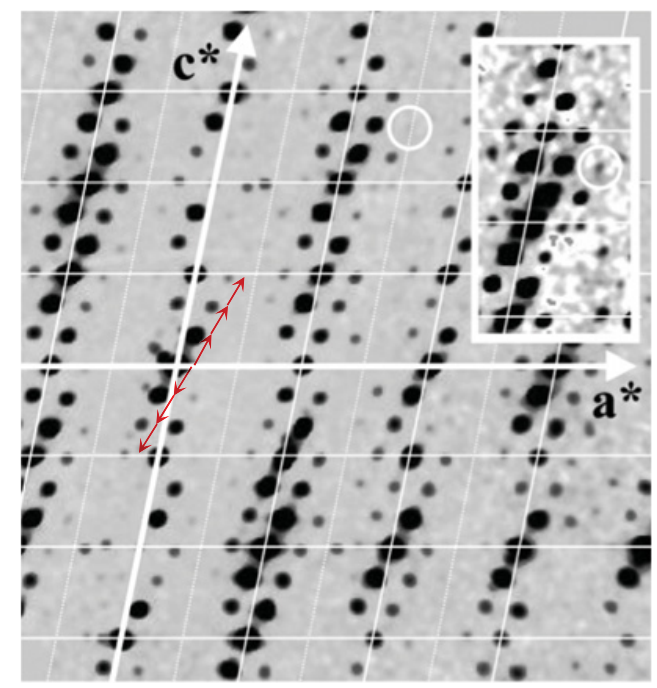

Figure 4. $(h, 0, l)$ diffraction plane of $\mathrm{Na}_{2} \mathrm{CO}_{3}$. The white grid represents the lattice of the main reflections. The additional (satellite) diffraction spots are regularly displaced from regular (main) spots by a q-vector (red arrows).

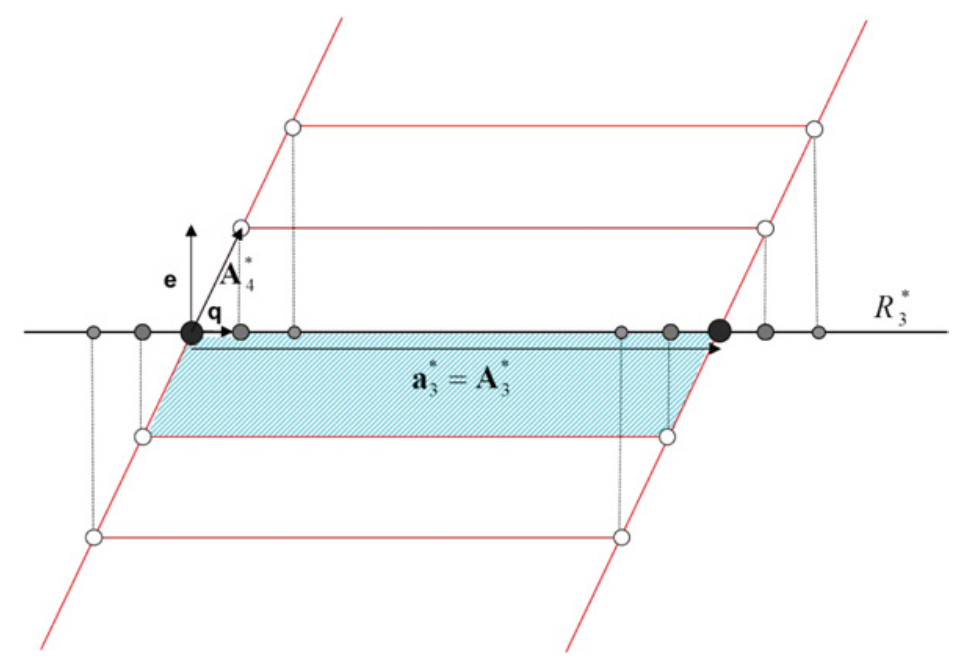

Figure 5. Introducing superspace in the reciprocal space: the vector $\mathbf{e}$ is perpendicular to the diffraction space $R_{3}^{*}$. Black spots are main reflections located at the nodes of the three-dimensional reciprocal lattice. Gray spots are satellite reflections. The new reciprocal lattice vector: $\mathbf{A}_{4}^{*}=\mathbf{q}+\mathbf{e}$. White spots are satellites projected to the new lattice vector; the blue area is a $(3+1) \mathrm{d}$ reciprocal cell describing main reflections as well as satellites.

nuclear density function fulfills the equation:

$$
\tilde{\rho}(\mathbf{r})=\tilde{\rho}\left(\mathbf{r}+\sum_{i=1}^{4} n_{i} \mathbf{A}_{i}\right)
$$




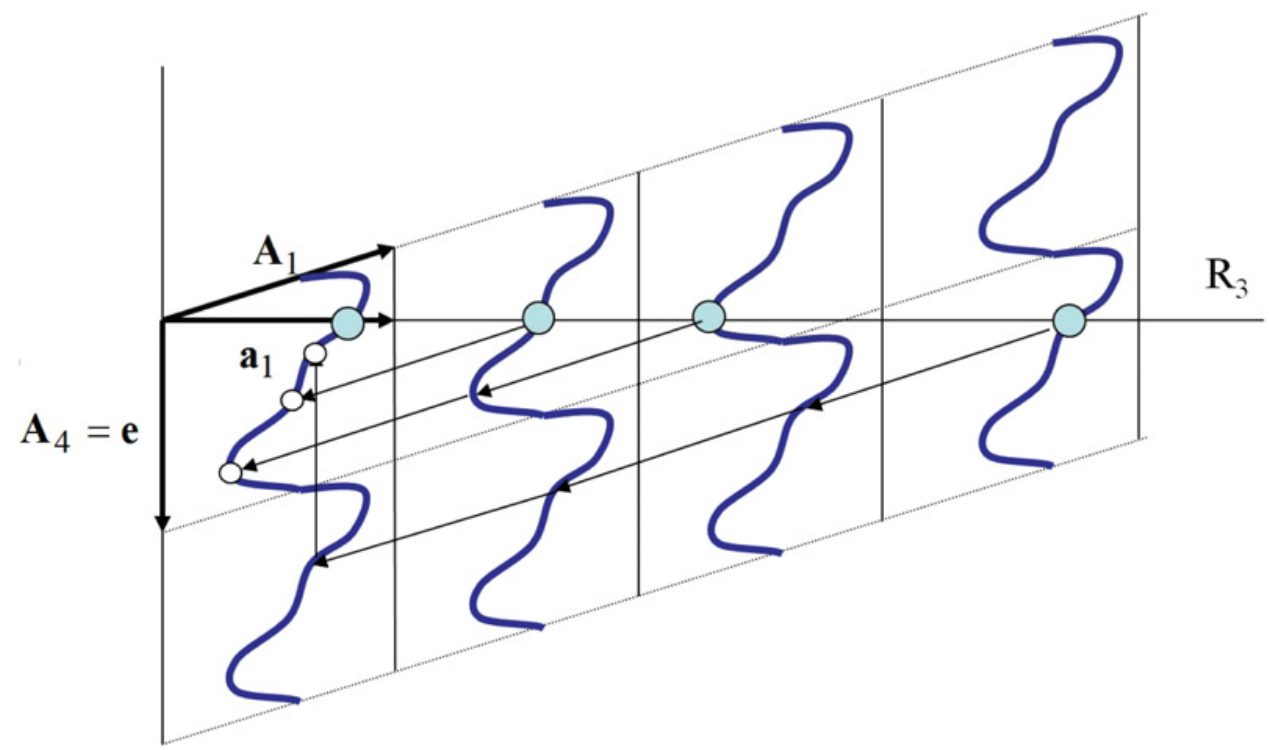

Figure 6. The positionally modulated structure in the $(3+1) \mathrm{d}$ superspace: the atomic positions are described by the modulation function defined in a $(3+1) d$ unit cell. This unit cell can be repeated by the $(3+1) \mathrm{d}$ translational symmetry to fill the whole $(3+1) \mathrm{d}$ superspace. Large blue points indicate intersections of the modulation function with $\mathrm{R}_{3}$ (the real space). These intersections define the crystal structure. Due to the translation symmetry, each such point can be transformed to the first unit cell (as white points).

where the lattice vectors $\mathbf{A}_{i}$ fulfil the equations:

$$
\mathbf{A}_{i}=\mathbf{a}_{i}-\left(\mathbf{a}_{i} \cdot \mathbf{q}\right) \mathbf{e} \quad i=1,2,3 \quad \mathbf{A}_{4}=\mathbf{e}
$$

as follows from orthogonality conditions between direct $\mathbf{A}_{i}$ and reciprocal $\mathbf{A}_{i}^{*}$ :

$$
\mathbf{A}_{i} \cdot \mathbf{A}_{j}^{*}=\delta_{i j}
$$

In the Fig. 6 the translation symmetry is demonstrated for positionally modulated structure. There is again a unit cell which contains structural information needed to generate whole modulated structure. The superspace approach allows generalization of standard structure tools such as Fourier and Patterson syntheses, structure solution and refinement techniques.

As the really observed $3 \mathrm{~d}$ diffraction pattern is a projection from an auxilliary $(3+1) \mathrm{d}$ diffraction pattern in superspace along the vector e, corresponding $3 \mathrm{~d}$ Fourier map which shows a nuclear density, is a section perpendicular to the vector e through a $(3+1)$ d periodic superspace map. Moving this section along the vector e gives sections (so-called t-sections) of the same modulated structure which differs only by the origin shift.

The $(3+1)$ d Fourier maps are calculated from structure factors according to the equation analogical to the Eq. (6). Structure factor amplitudes are based on observed intensities (Eq. (7)) while phases follow from the actual model. The sections, in which modulation of one atomic coordinate $x_{1}, x_{2}$ or $x_{3}$ is visualized as a function of $x_{4}$, are called de Wolff's sections. They play a crucial role in finding and completing modulation models for individual atoms in the structure. 


\subsection{Superspace symmetry}

Symmetry operation $\hat{S}$ in the superspace must keep the generalized electron density invariant:

$$
\tilde{\rho}(\hat{S} \mathbf{r})=\tilde{\rho}(\mathbf{r})
$$

A set of symmetry operations fulfilling this equation constitutes a superspace group. In analogy to $3 \mathrm{~d}$ structures, the superspace symmetry induces diffraction symmetry, and the whole diffraction pattern including satellite reflections follows the corresponding point group. Introducing symmetry in $(3+1) \mathrm{d}$ superspace does not mean that all possible fourdimensional space groups are available for crystal symmetry. There are some restrictions which considerably reduce the number of four dimensional space groups acceptable for description of modulated structures. Any superspace operation $\mathbf{R}$ has the following matrix form with respect to the given crystallographic base:

$$
\mathbf{R}=\left(\left[\begin{array}{c|c}
\boldsymbol{\Gamma}_{E} & \mathbf{0} \\
\hline \boldsymbol{\Gamma}_{M} & \boldsymbol{\Gamma}_{I}
\end{array}\right],\left[\begin{array}{c}
\mathbf{s}_{E} \\
\hline \mathbf{s}_{I}
\end{array}\right]\right)
$$

The left-upper part $\boldsymbol{\Gamma}_{E}$ is $3 \times 3$ matrix which represents proper or improper rotation in three dimensional space (known from $3 \mathrm{~d}$ space groups). The right-upper part is $3 \times$ 1 matrix (a column) of zeros. This restriction follows from the fact that superspace is based in a mathematical construction which uses an auxilliary vector e. This vector must stay perpendicular to the real three dimensional space when the symmetry operation is applied. The right-lower block $\Gamma_{I}$ is $1 \times 1$ matrix with value equal \pm 1 . Finally, the left-lower block $\Gamma_{M}$ is $1 \times 3$ matrix (row) fulfilling the equation:

$$
\mathbf{q} \Gamma_{E}-\Gamma_{I} \mathbf{q}=\Gamma_{M}
$$

This means that the rotation part of the symmetry operation is fully determined from the $3 \mathrm{~d}$ rotation part and modulation vector.

The translation part $\mathbf{s}_{E}$ follows from the corresponding $3 \mathrm{~d}$ space group. The only new (compared with standard crystallography) part is $\mathbf{s}_{I}$ which defines the translation component along the additional $4^{\text {th }}$ direction. Value of this component can induce systematic extinctions of satellite reflections. For example the mirror plane with its normal parallel to the c axis and $\Gamma_{I}=1$ causes extinctions as described in the Table 2 . In this table, the symbol used for symmetry elements consists from two parts, the first one defines the $3 \mathrm{~d}$ symbol and the second one the additional information about $\mathbf{s}_{I}$, which in our example is 0 and $\mathrm{s}$ for $\mathbf{s}_{I}$ equal 0 and $1 / 2$, respectively.

The superspace symbols are described in the International Tables for Crystallography (IT) vol. C [9]. The symbol consists of three parts - space group symbol, specification of the modulation vector and specification of the $\mathbf{s}_{I}$ components of the symmetry operations. The components of modulation vector are either restricted by the Eq. (27) to specific rational values or they can have general (irrational) value. Irrational components are specified by Greek letters $\alpha, \beta$ or $\gamma$. Example: Pmna $\left(\begin{array}{ll}0 & 1 / 2\end{array}\right) s 00$. Information available in the IT vol. $\mathrm{C}$ is limited, without a list of superspace group operations which restrict modulation functions of atoms located at special positions. Fortunately the list of symmetry operations for superspace groups up to $(3+3)$ d superspace as well as additional details about their standard settings is accessible from [10]. The symmetrical restrictions of modulated functions expressed as a combination of harmonic waves for $(3+1)$ superspace groups have been published [11] and they are automatically applied in the program Jana2006. 
Table 2. Systematic extinctions for $m(001)$.

\begin{tabular}{|l|c|c|}
\hline$\left(\boldsymbol{s}_{\mathbf{1}}, \boldsymbol{s}_{\mathbf{2}}, \boldsymbol{s}_{\mathbf{3}}, \boldsymbol{s}_{\mathbf{4}}\right)$ & Symmetry operation & Extinction condition \\
\hline$(0,0,0,0)$ & $\left(\begin{array}{c}m \\
0\end{array}\right)$ & - \\
\hline$(0,0,0,1 / 2)$ & $\left(\begin{array}{l}m \\
s\end{array}\right)$ & $m=2 n+1$ \\
\hline$(1 / 2,0,0,0)$ & $\left(\begin{array}{l}a \\
0\end{array}\right)$ & $h=2 n+1$ \\
\hline$(1 / 2,0,0,1 / 2)$ & $\left(\begin{array}{l}a \\
s\end{array}\right)$ & $h+m=2 n+1$ \\
\hline$(0,1 / 2,0,0)$ & $\left(\begin{array}{l}b \\
0\end{array}\right)$ & $k+m+1$ \\
\hline$(0,1 / 2,0,1 / 2)$ & $\left(\begin{array}{l}b \\
s\end{array}\right)$ & $h+k=2 n+1$ \\
\hline$(1 / 2,1 / 2,0,0)$ & $\left(\begin{array}{l}n \\
0\end{array}\right)$ & $h+k+m=2 n+1$ \\
\hline$(1 / 2,1 / 2,0,1 / 2)$ & $\left(\begin{array}{l}n \\
s\end{array}\right)$ & $k+1$ \\
\hline
\end{tabular}

\subsection{Basic modulation types}

Modulations in the crystal can affect all structural parameters i.e. atomic occupancies, positions, ADP, multipole coefficients or magnetic moments. Any structural parameter $p\left(x_{4}\right)$ due to the translation symmetry in the superspace can be expressed as a Fourier expansion:

$$
p\left(x_{4}\right)=p_{0}+\sum_{n}\left(p_{s, n} \sin 2 \pi n x_{4}+p_{c, n} \cos 2 \pi n x_{4}\right)
$$

where $p_{0}, p_{s, n}, p_{c, n}$ are expansion coefficients which are used to describe modulation wave. The number of used terms in the Eq. (28) depends on complexity of the modulation in crystal which is usually connected with the maximal number of observable satellite reflections. In the following a simple modulation models and their influence to the diffraction pattern is presented.

\subsubsection{Occupational modulation}

The simplest occupational modulation with only one harmonic wave can be expressed like:

$$
p\left(x_{4}\right)=p_{0}+p_{s, 1} \sin \left(2 \pi x_{4}\right)+p_{c, 1} \cos \left(2 \pi x_{4}\right)
$$

From the kinematical theory of diffraction it can be shown that such modulation will generate only first order satellites, as visualized in the Fig. 7(a). The corresponding de Wolff's section through the $(3+1)$ d Fourier map is shown in Fig. 7(b):

This simple harmonic model for occupation modulation is not very common in reallife examples. Usually the diffraction pattern also contains higher order satellites and the description of modulations needs more harmonic waves. In the limiting case the modulation takes step like character:

$$
\begin{array}{ll}
p\left(x_{4}\right)=1 & x_{4} \in\left\langle x_{4}^{0}-\frac{\Delta}{2}, x_{4}^{0}+\frac{\Delta}{2}\right\rangle \\
p\left(x_{4}\right)=0 & x_{4} \notin\left\langle x_{4}^{0}-\frac{\Delta}{2}, x_{4}^{0}+\frac{\Delta}{2}\right\rangle
\end{array}
$$

where $x_{4}^{0}$ and $\Delta$ define the center and length of the $x_{4}$ interval in which the atom is present. 


\section{JDN 22}

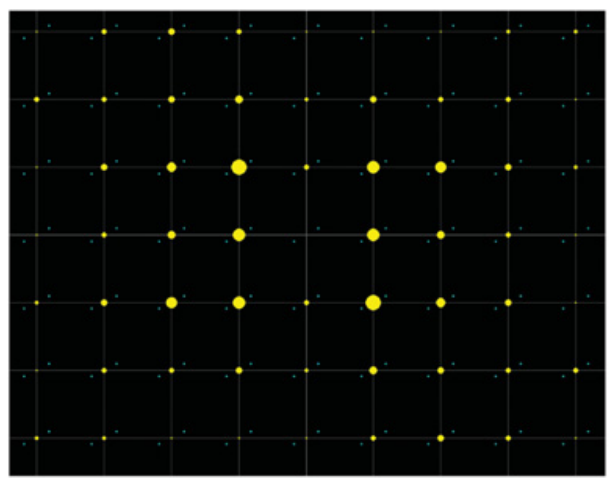

(a)

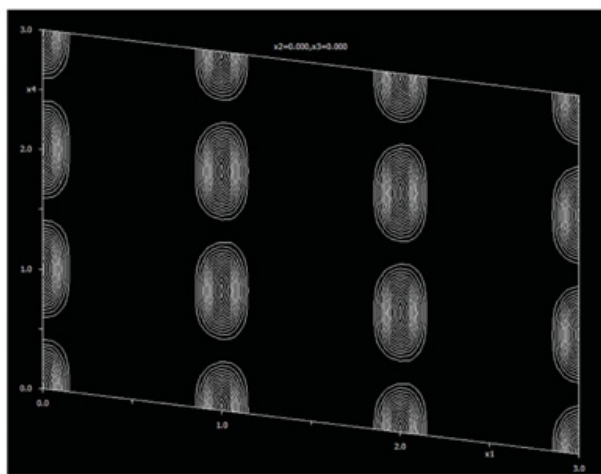

(b)

Figure 7. The simple harmonic occupational modulation: (a) diffraction pattern, (b) de Wolff's section.

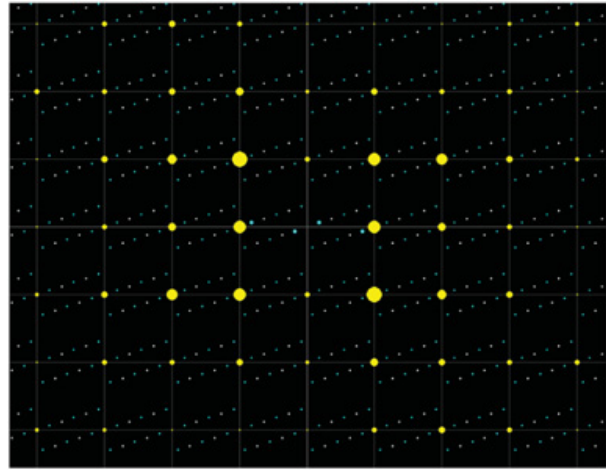

(a)

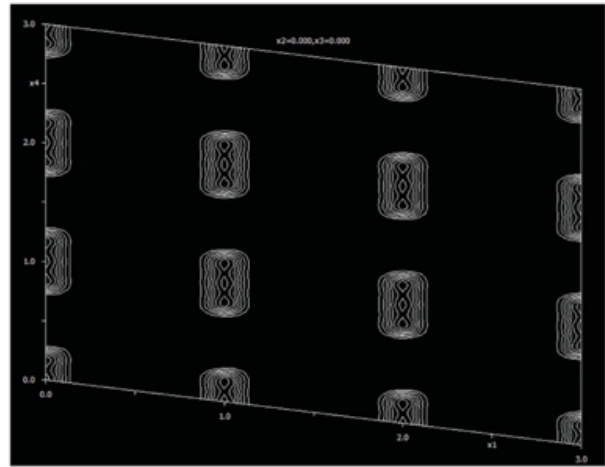

(b)

Figure 8. The step like occupational modulation (so-called crenel modulation): (a) diffraction pattern, (b) de Wolff's section.

As shown in the Fig. 8, the step like modulation leads to a high number of satellite reflections. Such a modulation is called crenel modulation [12]. Description of step-like modulation by harmonic waves would require high number of parameters. Instead of them we can use a crenel function (30) which has only two parameters - the center and the width.

\subsubsection{Positional modulation}

The diffraction patterns for weak and strong modulation amplitude are shown in the Figs. 9 and 10. On contrary to occupational modulation here one harmonic can generate higher satellites. With growing amplitude of the modulation the order and relative intensity of satellites increases.

\subsubsection{Composite structures}

These structures consist of two or more modulated subsystems each having its $(3+1) \mathrm{d}$ reciprocal lattice $\mathbf{A}_{i}^{* v} i=1,2,3,4$ ( $v$ denotes the subsystem) related to a reference lattice 


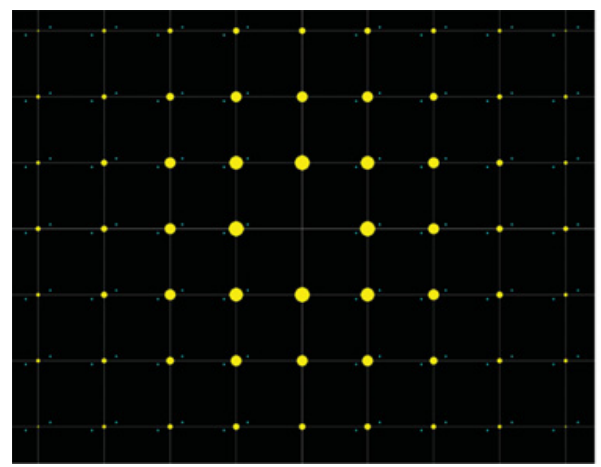

(a)

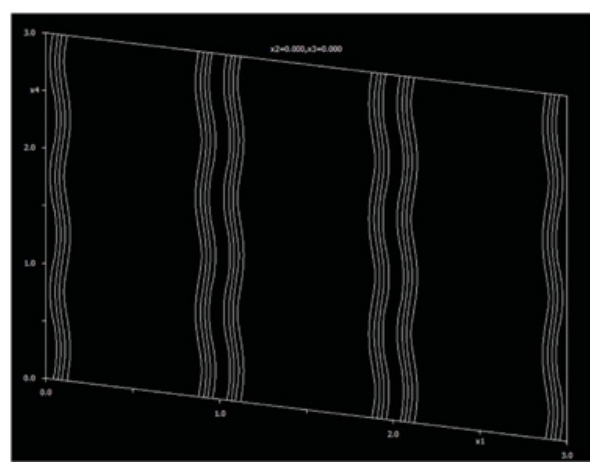

(b)

Figure 9. The weak positional modulation: (a) diffraction pattern, (b) de Wolff's section.

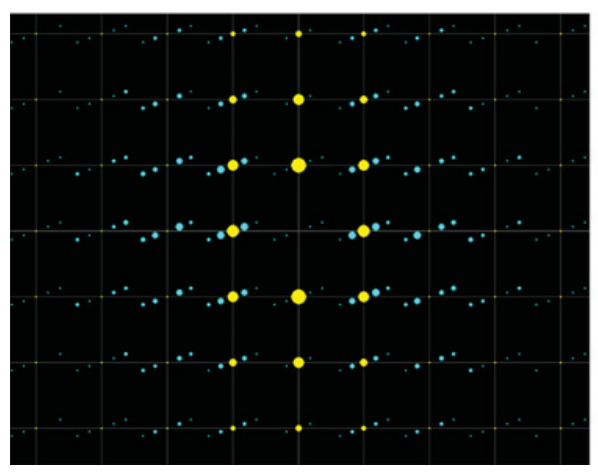

(a)

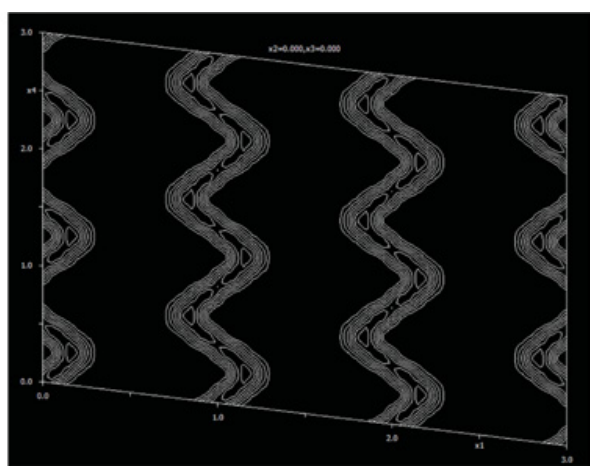

(b)

Figure 10. The strong positional modulation : (a) diffraction pattern, (b) de Wolff's section.

$\mathbf{A}_{i}^{*} i=1,2,3,4$ by the equation:

$$
\mathbf{A}_{i}^{* v}=\sum_{i=1}^{4} Z_{i j}^{v} \mathbf{A}_{j}^{*}
$$

where the $Z_{i j}^{v}$ is an integer matrix. Figure 11 shows a simple example of a composite structure made of two columns of atoms with different periodicity along the $\mathbf{a}_{1}^{1} \| \mathbf{a}_{1}^{2}$ direction, while the either parameters are identical: $\mathbf{a}_{2}^{1}=\mathbf{a}_{2}^{2}$ and $\mathbf{a}_{3}^{1}=\mathbf{a}_{3}^{2}$. In this example, the subsystems are not modulated and each of them makes a diffraction pattern which can be fully indexed by three indexes. We need four indexes, however, to index these patterns together.

If we choose the first subsystem as a reference subsystem, the $\mathrm{Z}$ matrices take form:

$$
\mathbf{Z}^{1}=\left[\begin{array}{llll}
1 & 0 & 0 & 0 \\
0 & 1 & 0 & 0 \\
0 & 0 & 1 & 0 \\
0 & 0 & 0 & 1
\end{array}\right] \quad \mathbf{Z}^{2}=\left[\begin{array}{llll}
0 & 0 & 0 & 1 \\
0 & 1 & 0 & 0 \\
0 & 0 & 1 & 0 \\
1 & 0 & 0 & 0
\end{array}\right]
$$




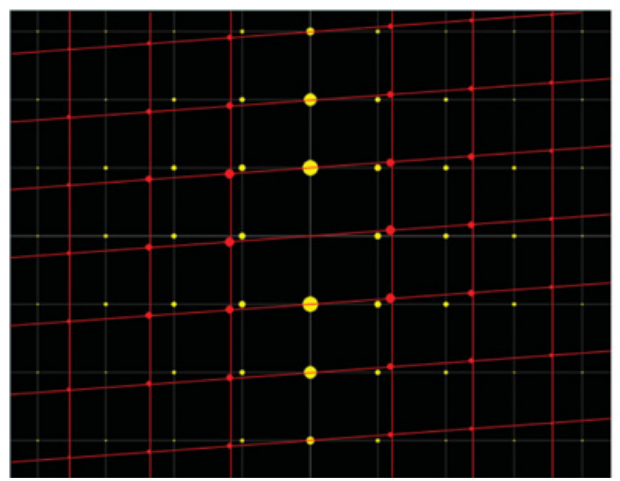

(a)

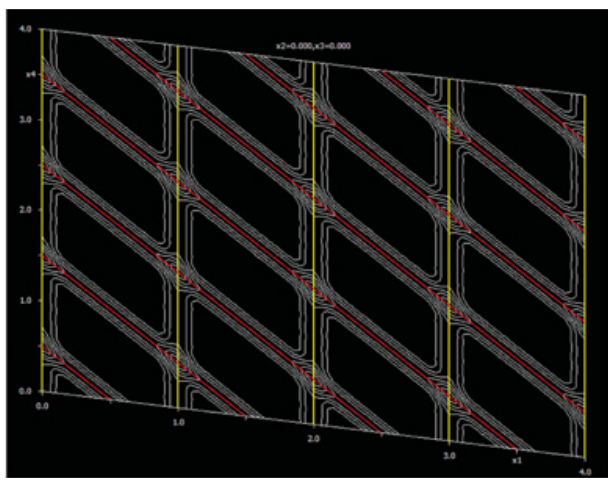

(b)

Figure 11. Composite structure composed of non-modulated subsystems: (a) diffraction pattern, (b) de Wolff's section.

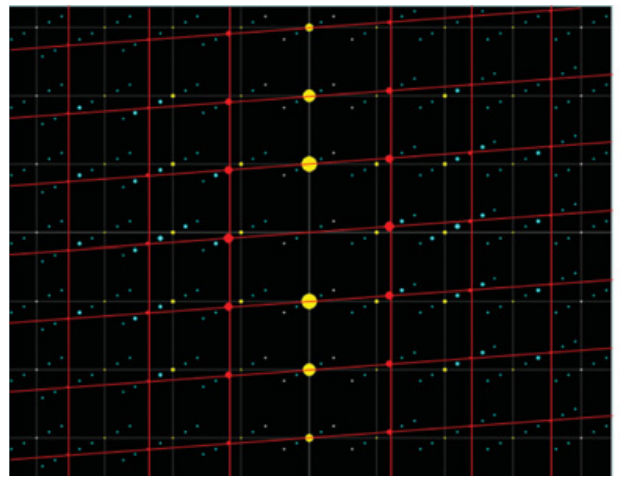

(a)

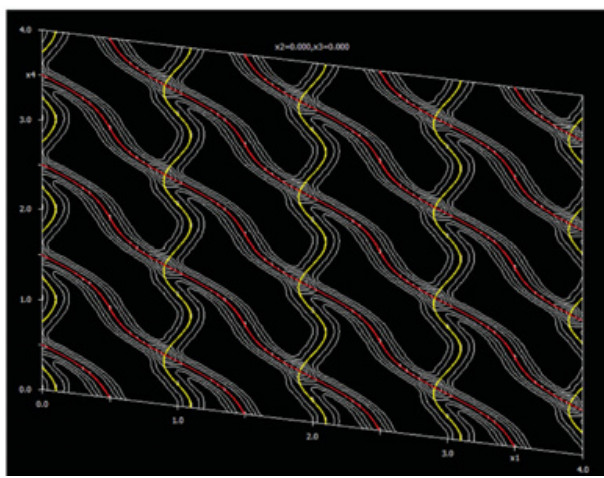

(b)

Figure 12. Composite structure composed of modulated subsystems: (a) diffraction pattern, (b) de Wolff's section.

Diffraction pattern of non-modulated composite crystal contains only main reflections of both subsystems. However, the interaction between both subsystems usually induces a mutual modulations as shown in the Fig. 12.

\subsection{Solution of modulated structures}

In many cases modulated structures can be solved in two steps. In the first step only main reflections are used to solve an average structure by standard methods. Such a structural model can show features like split atomic positions or unusually large ADP ellipsoids (see Fig. 13).

Such effects can help to predict type of modulations and recognize atoms which are strongly modulated. In the second step, the modulation waves are found by refinement from the small randomly chosen displacements.

However in case of strong modulations the second step or even the first step can fail because both the average structure and the initial modulation wave may be too far of the 


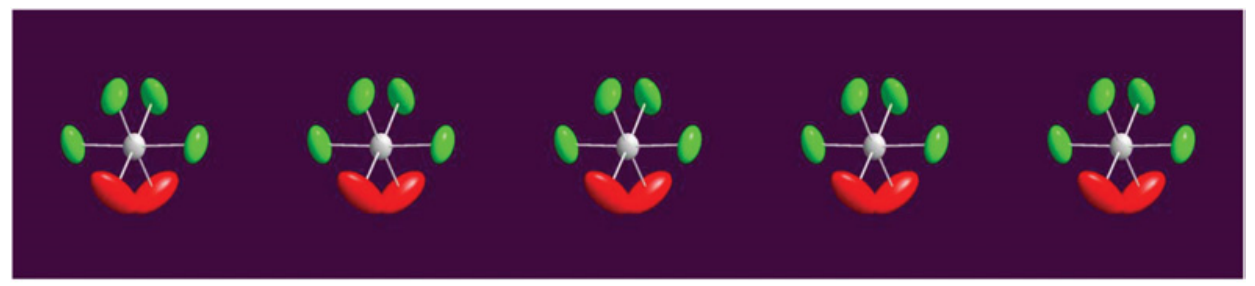

(a)

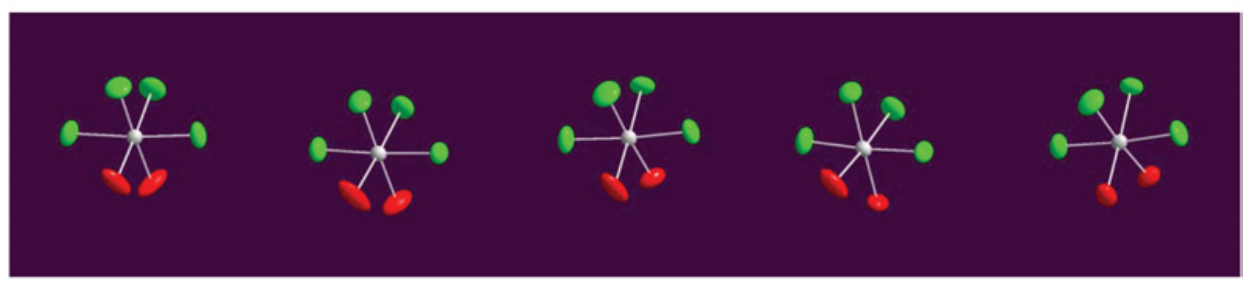

(b)

Figure 13. Anion $\mathrm{AsF}_{4}(\mathrm{OH})_{2}$ [16] (a) average structure calculated from main reflections; (b) modulated structure.

correct solution. As for the standard crystallography, refinement and Fourier methods only work for "almost finished" structures. This was the reason why the standard solution methods were generalized for modulated structures with the aim to solve them ab initio. The direct methods based on the Sayre equation were used for the development the program DIMS written by Fan Hai-fu [13, 14]. The heavy atom method for modulated crystals was developed by Steurer [15] and Petricek et al. [16]. However, these methods are not applicable to all cases.

The most promising method for $a b$ initio solution of strongly modulated structures is so-called charge flipping $[17,18]$. The method has been implemented into the program Superflip [19] and it is distributed together with program Jana2006. This method can solve modulated structures just in one step and starting modulation functions can be deduced directly from the output of the charge flipping, which is the nuclear density map in superspace.

With help of the modern diffractometer software and charge flipping, the steps like indexing, data collection, data processing and structure solution can be done almost routinely. However, it does not mean that complete solution of modulated structures is a routine job. Difficulties start after the solution step, where e.g. interpretation of the charge flipping output requires experience, manual work and analysis of Fourier sections. This interpretation is crucial for proper selection of modulation parameters and it can be specific for different modulated structures. This selection is important not only for refinement but especially for interpretation of results where we aim to understand the reasons why the structure is modulated.

Similarly as for twinned structures, the Jana cookbook contains several worked examples (5.1-5.5 and 7.1-7.3) covering typical cases of modulated structures. Moreover, everybody can participate in workshops which are offered regularly in the Jana web page for people interested in practical solution of modulated structures, as well as in bi-annual workshops organized by university in Bayreuth and in many other events focused to aperiodic crystallography. 


\section{References}

[1] G. Sheldrick, Acta Cryst. A64, 112 (2008)

[2] O.V. Dolomanov, L.J. Bourhis, R.J. Gildea, J.A.K. Howard, H. Puschmann, J. Appl. Cryst. 42, 339 (2009)

[3] P.W. Betteridge, J.R. Carruthers, R.I. Cooper, K. Prout, D.J. Watkin, J. Appl. Cryst. 36, 1487 (2003)

[4] V. Petříček, M. Dušek, L. Palatinus, Z. Kristallogr. 229, 345 (2014)

[5] A.L. Spek, PLATON, A Multipurpose Crystallographic Tool, Utrecht University, Utrecht, The Netherlands (2008)

[6] S. van Smaalen, Incommensurate Crystallography. (Oxford University Press, Oxford, 2007)

[7] T. Janssen, G. Chapuis, M. de Boissieu, Aperiodic Crystals. (Oxford University Press, Oxford, 2007)

[8] P.M. de Wollf, T. Janssen T., A. Janner, Acta Cryst. A37, 625 (1981)

[9] International Tables for Crystallography, Volume C, Ed. E. Prince (Kluwer Acad. Publ., Dordrecht, 2004)

[10] H.T. Stokes, B.J. Campbell, S. van Smaalen, Acta Cryst. A67, 45 (2011)

[11] V. Petříček, P. Coppens, Acta Cryst. A44, 1051 (1988)

[12] V. Petř́íček, A. van der Lee, M. Evain, Acta Cryst. A51, 529 (1995)

[13] Hao Quan, Liu Yi-wei, Fan Hai-fu, Acta Cryst. A43, 820 (1987)

[14] Fan Hai-fu, S. van Smaalen, E.J.W. Lam, P.T. Beurskens, Acta Cryst. A49, 704 (1993)

[15] W. Steurer, Acta Cryst. A43, 36 (1987)

[16] J. Peterková, M. Dušek V. Petříček, J. Loub, Acta Cryst. B54, 809 (1998)

[17] G. Oszlányi, A. Süto, Acta Cryst. A60, 134 (2004)

[18] L. Palatinus, Acta Cryst. A60, 604 (2004)

[19] L. Palatinus and G.Chapuis, J. Appl. Cryst. 40, 786 (2007) 